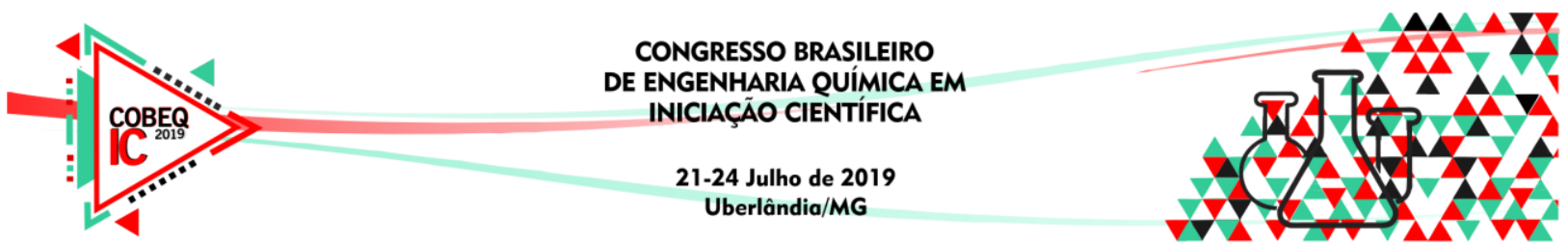

\title{
DESENVOLVIMENTO DE PROTÓTIPO DE SENSOR DE UMIDADE RESIDUAL APLICADO A PENEIRA VIBRATÓRIA
}

\author{
A. P. M. FREITAS ${ }^{1}$, A. H. IASBECK ${ }^{1}$, V. P. BARBOSA ${ }^{2}$, C. H. ATAÍDE ${ }^{2}$ e R. \\ GEDRAITE $^{2}$
}

${ }^{1}$ Universidade Federal de Uberlândia, Faculdade de Engenharia Mecânica

${ }^{2}$ Universidade Federal de Uberlândia, Faculdade de Engenharia Química E-mail para contato: rgedraite@ufu.br

\begin{abstract}
RESUMO - Neste trabalho foi estudado o protótipo de sistema de medição do teor de umidade dos sólidos retidos sobre a tela de separação de peneira vibratória industrial. Foi utilizado um sensor simples e de baixo custo para medir a umidade residual. O sinal capturado necessitou de condicionamento complementar para ser enviado ao sistema eletrônico de coleta de dados, uma vez que a coleta de dados se deu por amostragem. Os resultados obtidos se mostraram adequados e coerentes com o esperado, tendo o sistema de medição de umidade funcionado de forma satisfatória.
\end{abstract}

\section{INTRODUÇÃO}

A utilização de sistemas eletrônicos digitais em controle e automação de processos se iniciou, praticamente, com o próprio advento dos computadores digitais. Já no começo dos anos 60, instalações industriais introduziram o computador como importante dispositivo para controle. Durante aproximadamente 20 anos, tais aplicações concentraram-se na concepção de controle centralizado, ou seja, com o computador reunindo em um único processador eventualmente redundante - todas as suas tarefas (Seborg et al., 2005; Ogata, 2005). Atualmente, os modernos sistemas digitais de controle distribuídos, apresentam o recurso da distribuição do processamento das informações, viabilizados pelo baixo custo dos microprocessadores, permitindo assim uma confiabilidade similar àquela obtida pelos pioneiros sistemas analógicos convencionais, ao mesmo tempo em que se oferecia toda a potencialidade dos sistemas digitais, especialmente sua flexibilidade, conferida pelas funções reprogramáveis (Ronaes et al., 2009).

Em processos de perfuração de poços de petróleo, o controle de sólidos é de extrema importância devido a fatores operacionais, ambientais e econômicos. Os equipamentos utilizados em uma unidade típica de controle de sólidos para separação sólido-líquido são divididos em três grupos sequenciais: peneiras vibratórias, bateria de hidrociclones (desareiadores e dessiltadores) e centrífuga decantadora (Lobato et al., 2011). Tradicionalmente, o controle adequado e eficiente do funcionamento das peneiras vibratórias é feito de maneira simples, sem contemplar o emprego de modelos matemáticos que permitam predizer o comportamento das variáveis de processo de maior interesse para a operação, a saber: eficiência de separação e teor de umidade residual no material retido pela peneira. 


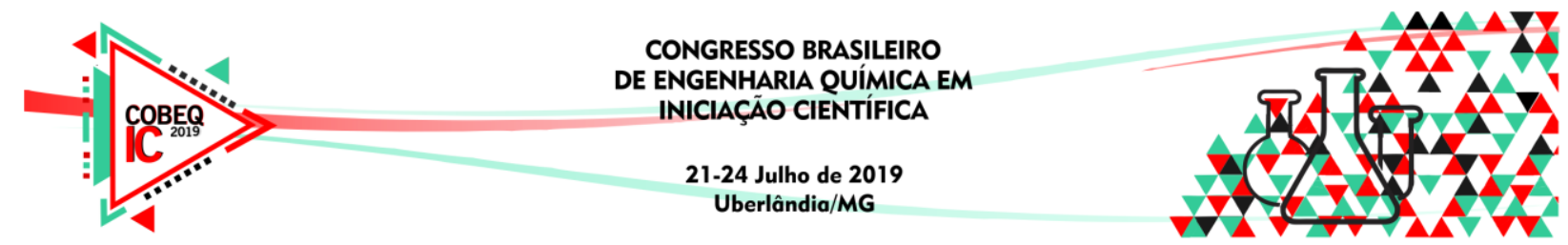

É importante ressaltar o fato de que há significativa dificuldade de se obter modelos fenomenológicos que sejam capazes de descrever o comportamento da mistura sólido-líquido em um processo de separação em peneira vibratória. Isso se deve principalmente ao grande número de variáveis envolvidas no processo, entre as quais podemos citar: abertura de tela, frequência de vibração (força-g), inclinação da tela de peneiramento, distribuição de tamanho de partícula, concentração de sólidos na mistura alimentada, interações partícula-partícula e líquido-partícula, entre outras.

Este trabalho tem como objetivo apresentar uma contribuição ao tema, abordando a implementação de sistema de medição do teor de umidade dos sólidos retidos. A verificação do teor de umidade do conteúdo que deixa a peneira permite que a eficiência deste equipamento seja analisada. Este trabalho teve como foco a avaliação da relação entre a intensidade da vibração do equipamento, determinada a partir da força-g a ele associada, e o teor de umidade do material de saída, que deve ser o menor possível.

\section{MATERIAIS E MÉTODOS}

Para medição da umidade do material de saída da peneira, foi utilizado o sensor de umidade de solo apresentado na Figura 1(a). Este é constituído por uma sonda resistiva, um módulo contendo um chip comparador LM393, que possibilita que a umidade do material seja relacionada a um sinal de tensão analógico gerado com range de $0 \mathrm{~V}$ a $5 \mathrm{~V}$ e dois jumpers $\mathrm{F}-\mathrm{F}$ (fêmea-fêmea) para conectar a sonda ao módulo. Sua leitura foi realizada por meio da utilização de uma placa de aquisição de dados, modelo NI USB-6211 da National Instruments $^{\mathrm{Tm}}$, apresentada na Figura 1(b), associada a um código computacional desenvolvido no aplicativo LabView $2013^{\mathrm{Tm}}$. O conversor analógico digital da NI USB-6211 possui resolução de 16 bits, assim têm-se 65536 faixas de valores para a conversão do sinal e um erro máximo de $0,000038 \mathrm{~V}$.

Figura 1 - (a) sensor de umidade de solo;(b) placa NI USB-6211 utilizados no trabalho

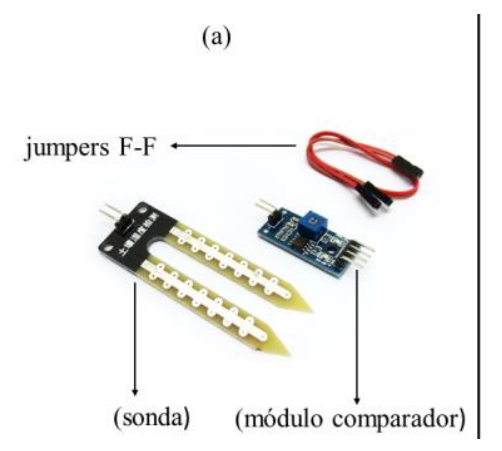

(b)

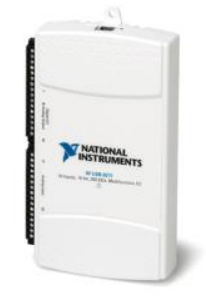

Para verificar a relação entre a intensidade da vibração da peneira e o teor de umidade do material na saída da mesma, foram realizados experimentos simples que consistiram em alimentar uma suspensão de areia em água - $10 \%$ em volume - à peneira vibratória em estudo, com a coleta eletrônica do valor do teor de umidade residual. Tal coleta consistiu em posicionar a sonda no material peneirado em torno de 30 segundos, retirar pelo mesmo tempo e posicionar novamente, para que, a cada posicionamento, uma nova amostra de material tivesse passado pela peneira. Assim, o sensor foi posicionado nove vezes para cada força-g, 


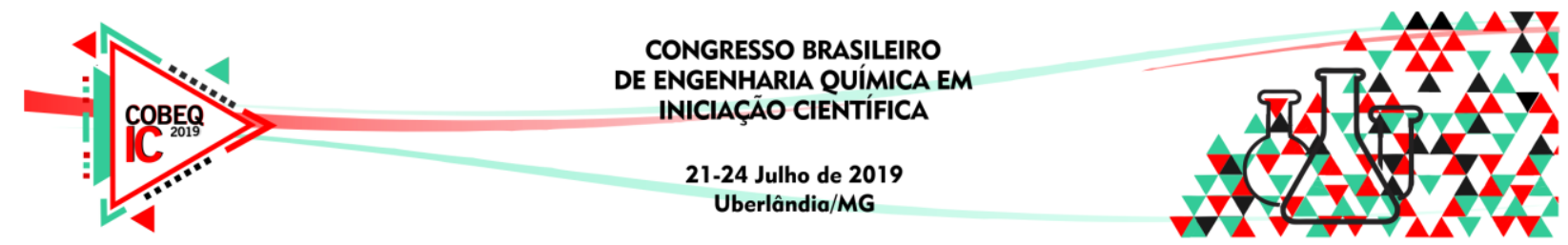

ou seja, foram analisadas nove amostras. O tempo de resposta do mesmo é relativamente alta, por isso uma exposição de apenas 30 segundos foi o suficiente para obter a média do sinal que representasse a umidade para cada força-g. O sinal do sensor de umidade foi aferido com base em amostras coletadas em Becker e colocadas para secar por 24 horas em estufa a $105^{\circ} \mathrm{C}$. Foi coletada uma amostra para cada valor de frequência de vibração.

\section{RESULTADOS OBTIDOS E DISCUSSÃO}

Na Figura 2(a) é apresentado o comportamento do sinal coletado na saída do sensor de umidade, em volts (corrente contínua), para a força-g de amplitude $3.3 \mathrm{G}$, em que apenas os pontos marcados em vermelho representam a umidade válida do material na saída da peneira. Estes podem ser mais bem observados na Figura 2(b). Importante destacar que cada conjunto de pontos representa a coleta automática de dados sobre uma mesma porção de material. $\mathrm{O}$ mesmo experimento foi realizado para as forças-g de amplitude $2 \mathrm{G}, 3 \mathrm{G}$ e $3.7 \mathrm{G}$.

E assim, foi possível representar os valores de tensão coletados em cada intervalo de amostragem por uma única amostra, fazendo a média de tensão sobre cada conjunto de pontos, conforme Figura 3(a).

Figura 2 - Umidade em Volts coletada a partir do sensor de umidade para a força $3.3 \mathrm{G} \mathrm{em}$ (a) experimento completo e (b) ênfase em apenas uma parte do experimento.

(a)

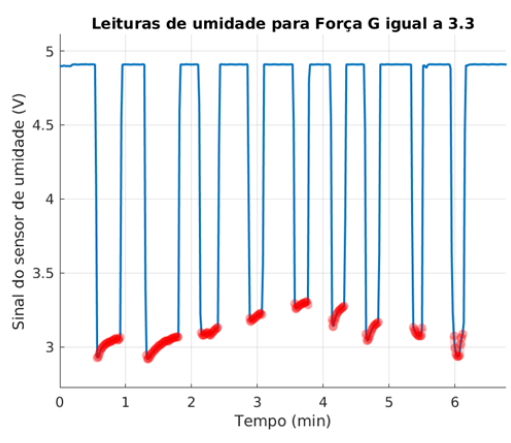

(b)

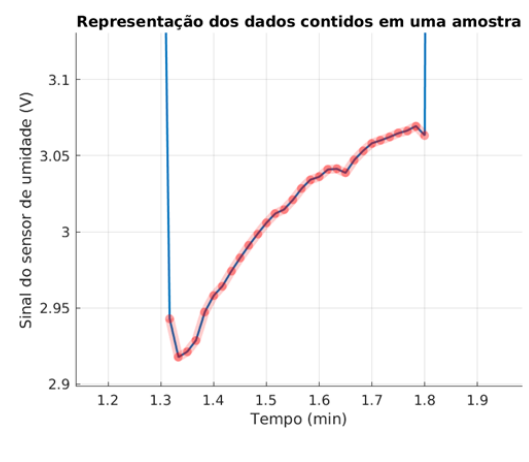

Para obtenção do modelo dinâmico que representasse adequadamente o comportamento do sinal medido, foi construído um conjunto de dados considerando todas as amostras coletadas em cada valor de força-g. Neste, foram concatenadas todas as amostras obtidas para cada valor de força-g, conforme apresentado na Figura 3(b). Com isso, é possível observar que à medida que a força-g aumenta, a tensão diminui. Com base na análise da folha de especificação técnica do sensor de umidade, foi possível concluir que à medida que o teor de umidade aumenta, a tensão em sua saída diminui. Portanto, com base nos resultados experimentais constatou-se que quanto maior a força-g, maior será o teor de umidade do material de saída da peneira.

Porém, como os valores de tensão gerados pelo sensor apresentam oscilações significativas, foi utilizado um filtro passa-baixa, dado pela Equação 1.

$$
y(t)=y(t-1)+\alpha[x(t)-y(t-1)
$$




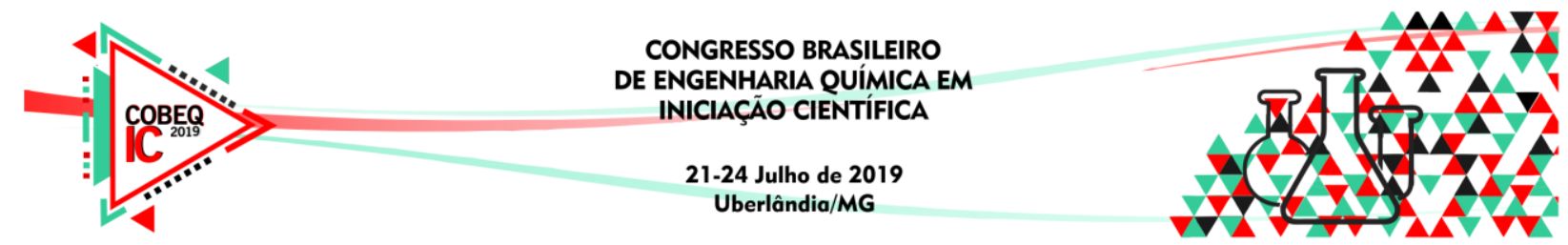

em que $x$ é o sinal de entrada do filtro (teor de umidade não filtrado), $y$ é o valor de sua saída (teor de umidade filtrado) e $\alpha$ é o fator de filtragem (Seborg et al., 2005) que foi obtido experimentalmente. Adotou $\alpha=0,4$, pois este não acarretou perda de informações do sinal original. A curva que relaciona o sinal original ao sinal filtrado pode ser visualizada na Figura 4(a).

Utilizando a ferramenta ident do MATLAB $^{\text {Tm}}$, foi ajustado um modelo dinâmico, apresentado na Equação 2, que representa a relação entre a amplitude da tensão média aferida na saída do sensor de umidade (diretamente relacionada ao teor de umidade) e a intensidade da vibração da peneira. Foi obtido um ajuste de $75 \%$ entre os dados gerados por meio do modelo e aqueles obtidos experimentalmente.

Figura 3 - Tensão média obtida a partir dos dados coletados em cada período de amostragem em (a) e (b) amostras coletadas para cada força-g.

(a)

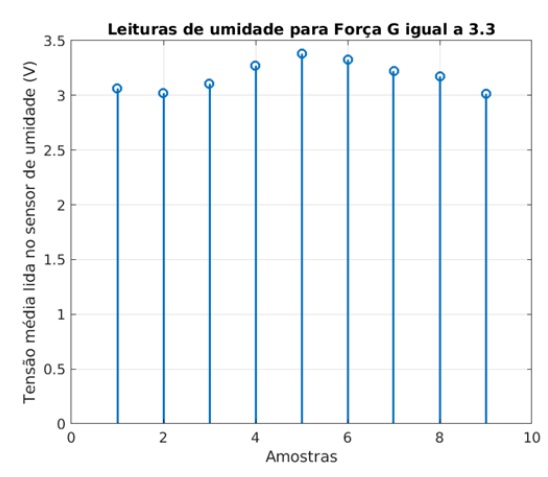

(b)

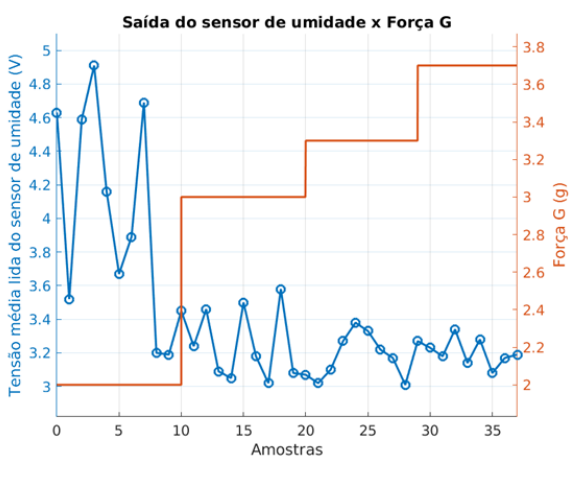

$G(s)=\frac{V(s)}{F g(s)}=\frac{0,97534}{(1+14,651 s)(1+2,6982 s)}$

Na Equação 2, $V(s)$ é a saída do sistema e representa a tensão média obtida por meio do sensor de umidade, e $F g(s)$ é a entrada do sistema representando a força-g. A comparação entre a saída estimada a partir do modelo dinâmico e a saída experimental é mostrada na Figura 4 (b).

É possível observar na Figura 4 (b) que o modelo obtido representa de maneira adequada e coerente o comportamento da tensão média na saída do sensor de umidade obtida experimentalmente. Assim, para melhor análise da natureza física do problema, foi necessário determinar uma função que permitisse representar adequadamente o teor de umidade obtido a partir do sinal de saída do sensor empregado. Para tal, foram comparados os valores de tensão média e o teor de umidade obtido experimentalmente com base em uma amostra coletadas em Becker e colocada para secar por 24 horas em estufa a $105^{\circ} \mathrm{C}$, para cada força-g. Estes dados podem ser observados na Tabela 1 e na Figura 5(a). 


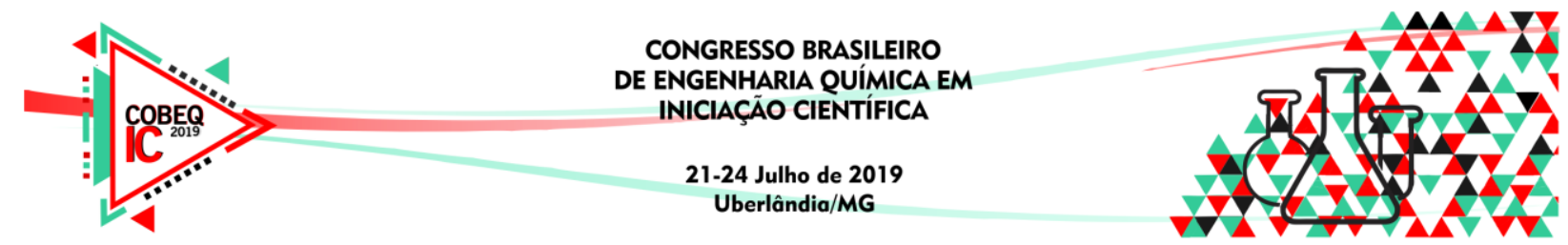

Figura 4 - Em (a) os sinais real e filtrado e em (b) saída real e a saída estimada a partir do modelo dinâmico obtido.

(a)

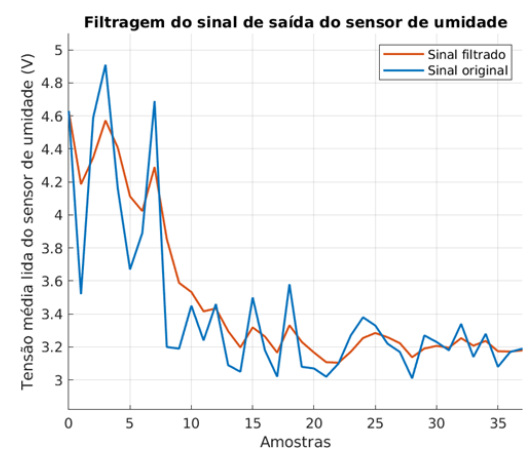

(b)

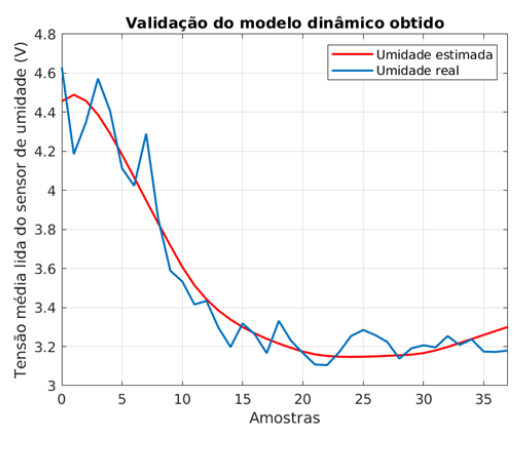

Tabela 1 - Relação de umidade em volts e em porcentagem para cada força-g.

\begin{tabular}{|c|c|c|}
\hline Força-g & $\begin{array}{c}\text { Sinal de saída } \\
\text { do sensor (V) }\end{array}$ & Umidade (\%) \\
\hline \hline 2,0 & 4,045 & 20,8518 \\
\hline 3,0 & 3,265 & 20,5664 \\
\hline 3,3 & 3,174 & 21,0794 \\
\hline 3,7 & 3,209 & 21,2283 \\
\hline
\end{tabular}

O sensor de umidade fornece alta tensão para teor de umidade baixo, sendo assim, na Figura 5(a) é possível notar que o ponto de coordenadas $(3,265 ; 20,5664)$ é discrepante, pois para uma tensão baixa espera-se uma umidade alta, com isso, tal ponto foi desconsiderado. É conhecido o comportamento linear entre o sinal do sensor e o teor de umidade, logo uma regressão linear foi realizada sobre os três pontos restantes. A reta resultante da regressão pode ser visualizada na Figura 5(b) e é dada pela Equação 3.

Figura 5 - Em (a) a relação entre a média de cada uma das amostras coletadas para cada valor de força-g e a tensão na saída do sensor. Em (b) a reta obtida a partir de uma regressão linear sobre os pontos obtidos.

(a)

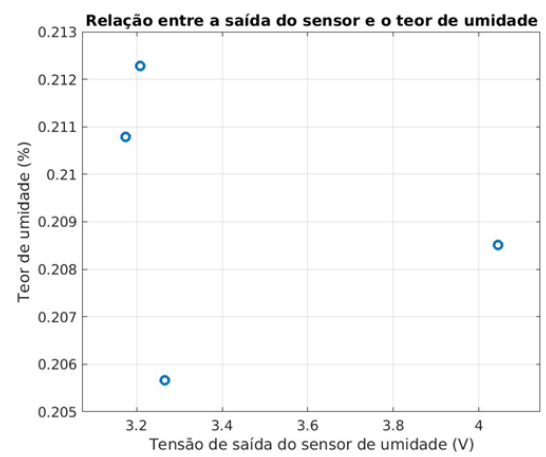

(b)

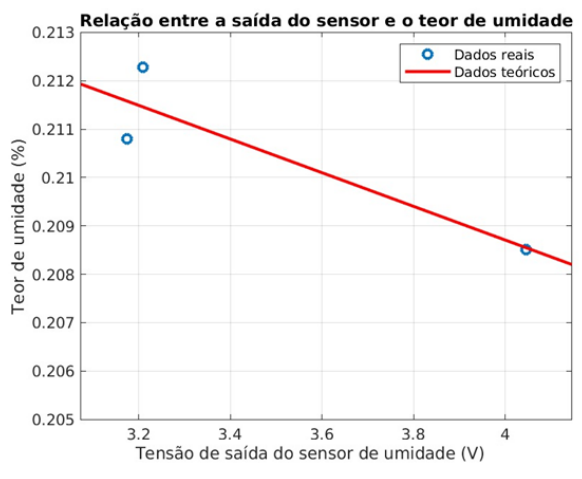




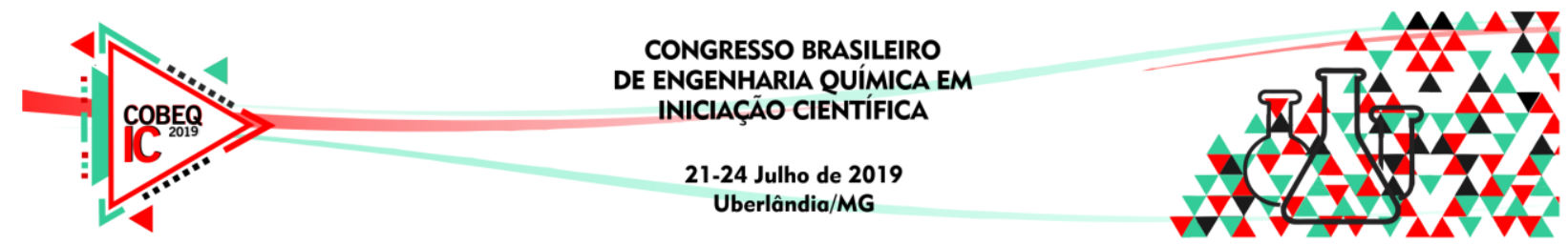

$H=-0,0035 V+0,2226$

onde $H$ é teor de umidade expresso em porcentagem e $V$ é o sinal da saída do sensor, em volts. Tal reta é de grande importância para conhecer a natureza física do problema estudado, pois sabe-se que a umidade é adimensional e a porcentagem representa a massa de fluido presente em uma amostra de sólido, mostrando o quão eficiente foi a separação sólidolíquido.

\section{CONCLUSÃO}

Com base na metodologia empregada neste trabalho foi possível estabelecer uma maneira simples e de baixo custo para medir o teor de umidade residual do material sólido retido na peneira. Adicionalmente, foi possível estabelecer um modelo matemático dinâmico para representar o comportamento do sinal medido, considerando as dificuldades da medição do teor de umidade em tempo real com o elemento sensor de baixo custo utilizado. Os próximos passos consistirão em melhorar a maneira de medir o teor de umidade e na implementação de malha de controle do teor de umidade acoplada com a malha de controle de força-g já existente.

\section{REFERÊNCIAS}

GUERREIRO, F. S.; GEDRAITE, R.; ATAIIDE, C. H. Residual moisture content and separation efficiency optimization in pilot-scale vibrating screen. Powder Technology, v. 287, p. 301-307, 2016.

LOBATO, F. S., OLIVEIRA-LOPES, L. C., GEDRAITE, R., NEIRO, S. M. S., MURATA, V. Identificação de modelos para unidades de recuperação do fluido de perfuração. In: IV Encontro Nacional de Hidráulica de Poços de Petróleo e Gás, Foz do Iguaçu - PR, 2011.

OGATA, K. Engenharia de Controle Moderno. Rio de Janeiro: Prentice Hall do Brasil, $3^{\mathrm{a}}$ edição, 2000.

RONAES, E., VIK, S. E., TAUGBOL, K., SJAHOLM, A.; KAAGESON-LOE, N. (2009). Remote real-time monitoring of particle size distribution in drilling fluids during drilling of a depleted HTHP reservoir. Proceedings of SPE/IADC, pp. 1-11, Manama, Bahrain, 26-28 October.

SEBORG, D. E., EDGAR, T. F. and MELLINCHAMP, D. A. (2005). Process Dynamics and Control. Wiley, 2nd Ed., USA.

VARGAS, I. K. G.; BARBOSA, V. P.; SILVA, M. E. D.; SILVA, R. M. GEDRAITE, R. Estudo experimental acerca da influência da vibração sobre o teor de umidade residual em material sólido granular utilizando protótipo de peneira vibratória. XX Jornada em Engenharia Química da Universidade Federal de Uberlândia, 2015. 